\section{S-275 USING METABOLOMICS TO TRACK BIOLOGICAL CLOCKS IN SHIFT WORK}

'Debra Skene. 'University of Surrey, United Kingdom

\subsection{6/OEM-2021-EPI.429}

Introduction Being able to monitor the timing of biological clocks in shift workers and assess the degree of circadian misalignment between these tissue clocks, the external environment and the mistimed behaviour (e.g. sleep and feeding) remains one of the challenges in shift work research. The circadian timing system compromises the light-entrainable central oscillator, located in the hypothalamic suprachiasmatic nuclei $(\mathrm{SCN})$, and peripheral clocks in almost all body tissues. Different shift work schedules result in changing light/dark; sleep/ wake; and feeding/fasting patterns that differentially affect the timing of these biological clocks.

Methods Using metabolic profiling (metabolomics) we have characterised time of day variation and the effect of sleep deprivation on the human metabolome1,2 providing a baseline for future metabolomics studies in shift workers.

Results Following simulated shift work, we found profound misalignment $(12 \mathrm{~h})$ of many plasma metabolite rhythms3. For $>90 \%$ of the metabolites, their 24-h rhythmicity was not locked to the central SCN circadian clock, rather, their rhythms aligned with the behavioural timing of the prior 3day simulated shift schedule.

Conclusions These findings provide a window onto metabolic pathways potentially involved in the elevated risk of metabolic disorders in shift work. Metabolic profiling provides a novel way to assess the effects of mistimed sleep/wake and feeding/ fasting schedules on metabolic pathways and investigate the underlying mechanisms linking metabolic disease, circadian misalignment and sleep deprivation.

1. Davies SK et al., Effect of sleep deprivation on the human metabolome. Proc Natl Acad Sci USA (2014) 111, 10761-10766; 2. Honma A et al., Effect of acute total sleep deprivation on plasma melatonin, cortisol and metabolite rhythms in females. Eur J Neurosci (2020) 51, 366-378; 3. Skene DJ et al., Separation of circadian- and behavior-driven metabolite rhythms in humans provides a window on peripheral oscillators and metabolism. Proc Natl Acad Sci USA (2018) 115, 7825-7830.

\section{S-278 INTRODUCTION TO THE CHALLENGES OF OCCUPATIONAL CODING}

${ }^{1}$ Cheryl Peters. ${ }^{1}$ University of Calgary, Canada

\subsection{6/OEM-2021-EPI.430}

Objective The objective of this presentation is to provide an introduction to the symposium entitled: 'A road map for artificial intelligence and occupational health in the 21st century.' Methods There are many important applications for industry and occupation codes in occupational hygiene and epidemiology. Many health studies collect job-related information in the form of free text (regarding a job title or tasks normally performed by a worker) which needs to be classified into a code in order to group similar workers together for analysis. Additionally, one of the standard tools for assigning exposure metrics to individuals in large studies is a job-exposure matrix (JEM) which is also linked to an industry code, an occupation code, and often both.
Results For the question of coding free-text into an appropriate code, for most of the history of occupational epidemiology, this has been done manually (and ideally by an expert $\operatorname{coder}(\mathrm{s}))$. This can be extremely time-consuming and has its own issues of reliability. For the question of JEMs, if they are created in one coding system, it can be difficult and subjective to re-code them to a system for use in a different country or year, for example (many coding systems are refined over time). There are many new and promising AI and/or machine learning applications and tools being developed around the world.

Conclusions This introductory talk will set the stage for the symposium, providing a background on the issues with occupational coding systems and code assignment in general, and how automatic coding systems could improve occupational epidemiology.

\section{S-301 SENSING AND SAMPLING FOR THE EXTERNAL EXPOSOME IN OCCUPATIONAL STUDIES}

${ }^{1}$ Miranda Loh, Eelco Kuijpers, Sander Ruiter, Susanne Steinle, Jan Mink, Koen Holgenelst, Peter Tromp, Ferry Jagers, Mark Cherrie, Vivi Schlünssen, Anne Mette Lund Würtz, Manolis Kogevinas, Barbara Harding, Gemma Castaño Vinyals, Anjoeka Pronk. 'Institute of Occupational Medicine, United Kingdom

\subsection{6/OEM-2021-EPI.431}

Introduction Characterizing the external exposome requires a combination of different measurement methods for multiple exposures.

Objectives We describe the development of an external exposome assessment system for the EPHOR study, which focuses on cohorts for assessing working life exposures, particularly one case study on shift work and one on respiratory health. The system will be implemented in a weeklong intensive nested study.

Methods A system of measurement using low-cost sensors, passive sampling methods, and a mobile phone app was developed and trialed. The low-cost sensor measures light, UV, noise, particulate matter, temperature and humidity in a high temporal resolution. Additionally, separate activity and heart rate monitors are added to the data stream. Passive sampling is used for a wide spectrum of chemical and biological agents. The phone app is used for asking a set of questions related to health and exposure factors on a daily basis. The low-cost sensor performance was compared to reference monitors in both a controlled laboratory setting (static) and in a free living setting (personal).

Results Initial tests of the low-cost sensor system indicates that correlations with reference monitors are high for controlled laboratory tests but more variable when used in personal monitoring. Additional calibration may be necessary throughout the project, especially for particulate matter, with the possibility of using the raw sensor data (particle numbers of various sizes) calculating mass concentration.

Conclusion A comprehensive protocol to measure the external exposome has been developed. Next steps include the development of external exposome profiles based on the collected data for use in exposure response analysis for various outcomes. It can be challenging to develop a one-size-fits-all external exposome system for exposome studies with different objectives. A flexible platform that can incorporate different sensors or measurement methods is an important feature of external exposome measurement systems. 